In Supramolecular Chemistry, 2016, VOL. 28, NOS. 9-10, 842-848, http://dx.doi.org/10.1080/10610278.2016.1174237 accepted manuscript

\title{
Multiple inclusion complex formation of protonated ellipticine with cucurbit[8]uril: Thermodynamics and fluorescence properties
}

\author{
Zsombor Miskolczy, ${ }^{\mathrm{a}}{ }^{\text {László Biczók }}{ }^{\mathrm{a}^{*}}$, István Jablonkai ${ }^{\mathrm{b}}$ \\ ${ }^{a}$ Institute of Materials and Environmental Chemistry, Research Centre for Natural \\ Sciences, Hungarian Academy of Sciences, P.O. Box 286, 1519 Budapest, Hungary \\ ${ }^{b}$ Institute of Organic Chemistry, Research Centre for Natural Sciences, Hungarian \\ Academy of Sciences, P.O. Box 286, 1519 Budapest, Hungary
}

This work was supported by the Hungarian Scientific Research Fund (OTKA) under Grant K104201, MedInProt Protein Science Research Synergy Program, and János Bolyai Research Scholarship of the Hungarian Academy of Sciences.

\footnotetext{
* Corresponding author. E-mail: biczok.laszlo@ttk.mta.hu
} 


\begin{abstract}
The encapsulation of protonated ellipticine $\left(\mathrm{EH}^{+}\right)$in the cavity of cucurbit[8]uril (CB8) was studied in water at $\mathrm{pH} 4$ with spectrophotometric, fluorescence spectroscopic and isothermal calorimetric measurements. The formation of three types of inclusion complexes was observed depending on the host and guest concentrations. Not only one but also two $\mathrm{EH}^{+}$was capable of

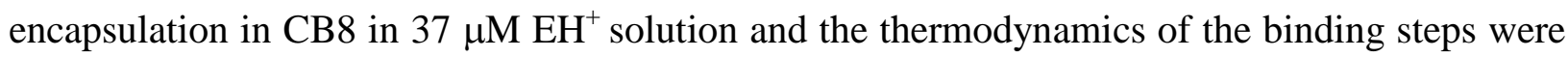
revealed. The produced very stable complexes showed markedly different absorption and fluorescence properties. When large excess of CB8 was employed in dilute $(0.49 \mu \mathrm{M}) \mathrm{EH}^{+}$ solution, sequential binding of two CB8 occurred to the monomer alkaloid bringing about a substantial alteration in the fluorescence decay kinetics. The driving force of the 1:2 guest:host complex formation was much lower than that of 1:1 encapsulation.
\end{abstract}

Key words: alkaloids, calorimetry, fluorescence decay, host-guest complex, binding affinity 


\section{Introduction}

The inclusion complex formation of cucurbiturils (CBn) with compounds of pharmaceutical importance has attracted widespread attention because of its great potential in drug delivery (1-4) and development of sensitive analytical methods (5-8). These macrocyclic hosts are nontoxic and capable of traversing the cell membrane (9-11). The confinement of drugs in CBn cavity not only enhances the solubility $(12,13)$ and thermal stability (14), but also hinders the decomposition $(15,16)$. For example, the nucleophilic addition reaction and photooxidation of sanguinarine, a biologically active natural benzophenanthridine alkaloid, are inhibited by the embedment in cucurbit[7]uril (CB7) macrocycle (17). The microenvironment-sensitive fluorescent properties of berberine were used to examine the encapsulation and dissociation kinetics with CBn cavitands $(18,19)$.

Due to its anticancer (20) and antimalarial (21) activity, ellipticine (E) has been extensively investigated, and the intricate mechanism of its biological effect has been revealed (22). E has a very low water solubility of $\sim 6.2 \times 10^{-7} \mathrm{M}$ at neutral $\mathrm{pH}(23,24)$, but more than 3 orders of magnitudes higher concentration can be the reached upon the protonation of the pyridine moiety. The conjugate acid has a $\mathrm{pK}_{\mathrm{a}}$ value of $7.4 \pm 0.1$ in $0.025 \mathrm{M}$ buffers (25). The fluorescent behavior of $\mathrm{E}$ was examined in organic solvents of a wide range of polarities and hydrogen bonding capabilities (26). The effect of interaction between the $\mathrm{NH}$ group of $\mathrm{E}$ and hydrogen bond acceptors on the absorption and fluorescence characteristics was revealed. Photoinduced deprotonation was found in acetonitrile only with a very strong base, 1,8diazabicyclo[5.4.0] undec-7-ene (DBU), due to the extremely weak acidity of the NH moiety of E even in the singlet-excited state (27). Therefore, photoinitiated tautomerization via intramolecular proton transfer from the pyrrolic $\mathrm{NH}$ to the nitrogen of the pyridine ring cannot occur. The lack 
of such a tautomerization was proved by the analogous fluorescence properties of $\mathrm{E}$ and its 6methyl derivative (ME), which does not contain any dissociable hydrogen $(28,29)$.

The encapsulation of the protonated form of ellipticine $\left(\mathrm{EH}^{+}\right)$in cucurbiturils has been examined, and only 1:1 complex formation was reported with both CB7 and cucurbit[8]uril (CB8) despite the substantial change of the fluorescence decay parameters upon gradual increase of host concentration (30). The main goals of the present studies were to understand the CB8 concentration dependence of the kinetics of $\mathrm{EH}^{+}$fluorescence and to identify the various fluorescent species. We reveal how the amounts of $\mathrm{EH}^{+}$and $\mathrm{CB} 8$ in the solutions affect the stoichiometry and thermodynamics of inclusion complex formation. The formulae of the investigated compounds are presented in Figure 1.

\section{Experimental}

Ellipticine ( $\geq 99 \%$ by HPLC, Fluka) was used as received. High-purity CB8 was kindly provided by Dr Anthony I. Day (University of New South Wales, Canberra, Australia). Experiments were performed in double-distilled water at $\mathrm{pH}$ 4. Slightly more than stoichiometric amount of concentrated $\mathrm{HCl}$ aqueous solution was added to ellipticine in ethanol. The solvent and the excess of $\mathrm{HCl}$ were evaporated. $\mathrm{EH}^{+} \mathrm{Cl}^{-}$salt prepared thereby was dissolved in $10^{-4} \mathrm{M} \mathrm{HCl}$ aqueous solution. The UV-visible absorption spectra were measured on an Agilent Technologies Cary60 spectrophotometer. Corrected fluorescence spectra were recorded on a Jobin-Yvon Fluoromax-4 photoncounting spectrofluorometer. No photodecomposition occurred when $\mathrm{EH}^{+}$ aqueous solutions were irradiated in the sample holder of the spectrofluorometer in the presence and absence of CB8. Fluorescence decays were collected with time-correlated single-photon counting technique using the previously described instrument (31). The results of spectrophotometric and fluorescence titrations were analysed with homemade programs written 
in MATLAB 7.9 analogously to that reported in a former paper (31). Starting with the initial estimates of the binding constant, the concentrations of the various species were calculated as numerical solutions of the mass balance equations and the definition of the binding constants. Then, the fluorescence intensity or absorbance values were calculated, and the iterations were repeated until the best fit was achieved. Isothermal titration calorimetry was carried out with a VP-ITC (MicroCal) instrument at $298 \mathrm{~K}$ as described (19).

\section{Results}

\subsection{Inclusion complex formation with $\mathrm{CB} 8$ at low $\mathrm{EH}^{+}$concentration}

To avoid dimerization, $0.49 \mu \mathrm{M}$ total protonated ellipticine concentration was used in the study of the interaction with CB8. Calculations using the recently published dimerization constant $\left((1.4 \pm 0.3) \times 10^{4} \mathrm{M}^{-1}\right)$ showed that less than $2 \%$ of the alkaloid molecules are associated in such a dilute solution (32). Gradual addition of $\mathrm{CB} 8$ to $\mathrm{EH}^{+}$solution at $\mathrm{pH} 4$ brought about an intensity enhancement and a hypsochromic shift in the fluorescence spectrum indicating complex formation (Figure 2A). The plot of the intensity at 530 and $560 \mathrm{~nm}$ as a function of CB8 concentration exhibits two distinct domains. The steep initial rise is followed by a much slower intensity enhancement suggesting sequential binding of two CB8 cavitands. The equilibrium constants are defined as

$$
\begin{aligned}
& K_{11}=\frac{\left[E H^{+}-C B 8\right]}{\left[E H^{+}\right][C B 8]} \\
& K_{12}=\frac{\left[E H^{+}-(C B 8)_{2}\right]}{\left[E H^{+}-C B 8\right][C B 8]}
\end{aligned}
$$

The results presented in Figure $2 \mathrm{~B}$ were analysed by a previously described homemade MATLAB 7.9 program (31). The nonlinear fit provided $K_{11}=(1.6 \pm 0.2) \times 10^{6} \mathrm{M}^{-1}$ and $K_{12}=$ $(5 \pm 4) \times 10^{3} \mathrm{M}^{-1}$, whereas the fluorescence efficiency at $560 \mathrm{~nm}$ was about 5-fold larger for 1:2 
$\mathrm{EH}^{+}-(\mathrm{CB} 8)_{2}$ complex than that of $1: 1 \mathrm{EH}^{+}-\mathrm{CB} 8$ complex. The microenvironment of the guest is less polar in $\mathrm{EH}^{+}-(\mathrm{CB} 8)_{2}$ than in water analogously to previous findings (33). Therefore, blueshift is observed in the fluorescence spectrum with the increase of CB8 concentration. Timeresolved fluorescence measurements at $590 \mathrm{~nm}$ corroborated the formation of two types of inclusion complexes. As expected, the amplitude of the dimer fluorescence was negligible $(<$ $2 \%$ ) in $0.49 \mu \mathrm{M} \mathrm{EH}{ }^{+}$solution. The fluorescence decays could be fitted by a triple-exponential function:

$$
I(t)=a_{1} \exp \left(-\frac{t}{\tau_{1}}\right)+a_{2} \exp \left(-\frac{t}{\tau_{2}}\right)+a_{3} \exp \left(-\frac{t}{\tau_{3}}\right)
$$

where $a_{i}$ stands for the amplitudes and $\tau_{i}$ denotes the lifetimes. Figure 3 shows the variation of the amplitude fractions $\left(\mathrm{a}_{\mathrm{i}} / \Sigma \mathrm{a}_{\mathrm{i}}\right)$ in the presence of various amounts of CB8. The monomer fluorescence of $2.0 \mathrm{~ns}$ lifetime vanished in the presence of large CB8 excess and the concomitant emergence of a $3.8 \mathrm{~ns}$ lifetime component was observed, which was assigned to singlet-excited $\mathrm{EH}^{+}-\mathrm{CB} 8$. The amplitude of this emission $\left(\mathrm{a}_{2}\right)$ reached a maximum around $7 \mu \mathrm{M}$ CB8 concentration and then declined due to the progressive strengthening of a third component of 16.6 ns lifetime. The longest-lived fluorescence was attributed to $\mathrm{EH}^{+}-(\mathrm{CB} 8)_{2}$ because its amplitude $\left(\mathrm{a}_{3}\right)$ grew at the expense of $\mathrm{a}_{2}$ at high CB8 concentrations. The substantial difference in the initial slope of $\mathrm{a}_{2}$ and $\mathrm{a}_{3}$ increase is in accordance with the more than two orders of magnitude larger equilibrium constant of $\mathrm{EH}^{+}-\mathrm{CB} 8$ formation compared to that of the binding of the second CB8.

To reveal the thermodynamics of $\mathrm{EH}^{+}$inclusion in $\mathrm{CB} 8$, the fluorescence titrations displayed in Figure 2 were repeated at various temperatures (T). The van't Hoff plot of the derived $K_{11}$ binding constant is presented in Figure 4. From the slope and intercept, $\Delta \mathrm{H}_{11}=-20 \pm 3 \mathrm{~kJ} \mathrm{~mol}^{-1}$ and $\Delta \mathrm{S}_{11}=51 \pm 7 \mathrm{~J} \mathrm{~mol}^{-1} \mathrm{~K}^{-1}$ were calculated for the enthalpy and 
entropy change in 1:1 host-guest complex formation. The thermodynamic parameters of CB8 association with $\mathrm{EH}^{+}-\mathrm{CB} 8$ could not be obtained due to the substantial uncertainty of $K_{12}$.

\subsection{Binding to $\mathrm{CB} 8$ at high $\mathrm{EH}^{+}$concentration}

Intriguing absorption and fluorescence characteristics was observed in CB8 solutions containing $37 \mu \mathrm{M}$ total $\mathrm{EH}^{+}$concentration. Under this condition, $38 \%$ of alkaloid molecules are dimerized in the absence of CB8 on the basis of the recently reported association constant of $\left(\left(K_{D}=1.4 \pm\right.\right.$ $0.3) \times 10^{4} \mathrm{M}^{-1}$ ) (32). Figure 5 displays the change of the absorption spectra and the absorbance at $327 \mathrm{~nm}$ upon increase of the amount of CB8 in the solution. The experimental data demonstrate that different species dominate at 22 and $97 \mu \mathrm{M}$ CB8 concentrations. This conclusion was corroborated by the results of fluorescence titration. (Figure 6) The apparent blue shift of the fluorescence maximum in the presence of $97 \mu \mathrm{M}$ CB8 originated from the dissociation of the $\left(\mathrm{EH}^{+}\right)_{2}$ dimer upon complex formation with CB8. To identify the binding processes, fluorescence decay measurements were performed at $530 \mathrm{~nm}$. The biexponential fluorescence intensity versus time profile arising from monomer and dimer $\mathrm{EH}^{+}$became triple exponential in the presence of $\mathrm{CB} 8$. Below $30 \mu \mathrm{M}$ CB8 concentration, lifetimes of 2.0, 3.8, and 7.5 ns were found. Upon further addition of $\mathrm{CB} 8, \mathrm{EH}^{+}$emission vanished, and a very weak emission of 16.6 ns lifetime emerged. The 2.0, 3.8 and 16.6 ns lifetimes have been ascribed to monomer $\mathrm{EH}^{+}$, $\mathrm{EH}^{+}-\mathrm{CB} 8$, and $\mathrm{EH}^{+}-(\mathrm{CB} 8)_{2}$ fluorescence, respectively. (vide supra) These assignments are corroborated by the variation of the amplitude fractions displayed in Figure 7.

The intensity of $2.0 \mathrm{~ns}$ lifetime component progressively diminishes and disappears above $30 \mu \mathrm{M}$ CB8 concentration because all $\mathrm{EH}^{+}$ions are encapsulated. This trend is accompanied by the parallel growth of the amplitude fraction of $\mathrm{EH}^{+}-\mathrm{CB} 8$ emission $\left(\tau_{2}=3.8\right.$ ns), which dominates at high $\mathrm{CB} 8$ concentrations. The $\mathrm{EH}^{+}-(\mathrm{CB} 8)_{2}$ fluorescence component 
$\left(\tau_{3}=16.6 \mathrm{~ns}\right)$ is much weaker than at low $\mathrm{EH}^{+}$concentration because of the smaller molar excess of CB8. The amplitude of $7.5 \mathrm{~ns}$ emission goes through a maximum as a function of CB8 concentration implying the formation of $\left(\mathrm{EH}^{+}\right)_{2}-\mathrm{CB} 8$ and its conversion into $\mathrm{EH}^{+}-\mathrm{CB} 8$. The confinement in CB8 insignificantly affects the fluorescence lifetime of $\left(\mathrm{EH}^{+}\right)_{2}$. No evidence was found for 2:2 binding. The Job plot of the absorbance change is presented in Figure S1 in Supporting Information. Among the experimentally detected species, the following association equilibriums are possible: (Figure 8)

Because the fluorescence decay traces showed very small amounts of $\mathrm{EH}^{+}-(\mathrm{CB})_{2}$ in 37 $\mu \mathrm{M} \mathrm{EH}{ }^{+}$solution over the entire CB8 concentration range, its formation was neglected in the evaluation of the experimental data presented in Figures 5 and 6. Our goal was to reveal the binding equilibriums resulting in $\left(\mathrm{EH}^{+}\right)_{2}-\mathrm{CB} 8$. This ternary complex may be produced (i) by consecutive binding of two $\mathrm{EH}^{+}$in $\mathrm{CB} 8$, (ii) by direct inclusion of $\left(\mathrm{EH}^{+}\right)_{2}$ dimer or (iii) both processes may take place. First, we assumed that consecutive binding of two $\mathrm{EH}^{+}$occurs. The reaction between $\left(\mathrm{EH}^{+}\right)_{2}$ and $\mathrm{CB} 8$ was eliminated and the experimental data were fitted with a MATLAB program developed on the basis of the remaining equilibriums. $K_{11}$ was known from

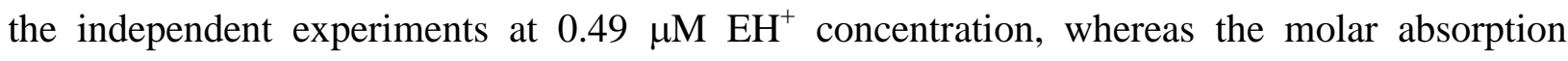
coefficient ratio at $327 \mathrm{~nm}\left(\varepsilon\left(\left(\mathrm{EH}^{+}\right)_{2}\right) / \varepsilon\left(\mathrm{EH}^{+}\right)=3.52\right)$ and the relative fluorescence efficiency at $530 \mathrm{~nm}\left(f\left(\left(\mathrm{EH}^{+}\right)_{2}\right) / f\left(\mathrm{EH}^{+}\right)=2.10\right)$ for $\left(\mathrm{EH}^{+}\right)_{2}$ and $\mathrm{EH}^{+}$as well as the association constant of $\left(\mathrm{EH}^{+}\right)_{2}$ dimer formation, $K_{D}$ were taken from measurements carried out in the absence of CB8. The nonlinear regression analysis of the spectrophotometric and fluorescence titration data at 37 $\mu \mathrm{M} \mathrm{EH}^{+}$concentration gave $K_{21}=(4.2 \pm 0.8) \times 10^{4} \mathrm{M}^{-1}$ and the computed functions matched the experimental data well (insets to Figures 5 and 6). 
In the second step, we assumed that $\left(\mathrm{EH}^{+}\right)_{2}-\mathrm{CB} 8$ is formed only in the association of $\left(\mathrm{EH}^{+}\right)_{2}$ with CB8. Thus, the complex formation between $\mathrm{EH}^{+}$and $\mathrm{EH}^{+}-\mathrm{CB} 8$ was eliminated. Excellent fit was obtained, and $K_{D l}=(5.2 \pm 0.95) \times 10^{6} \mathrm{M}^{-1}$ was found for the equilibrium constant of $\left(\mathrm{EH}^{+}\right)_{2}$ dimer confinement in CB8. This $K_{D 1}$ value corresponded to that calculated using $K_{21}, K_{11}$, and $K_{D}$ values given in Table 1 by the relationship

$$
K_{D 1}=K_{21} K_{11} / K_{D}
$$

Supporting Information shows the derivation of eq 4. This relationship is valid if the cycle of equilibriums shown in Figure 8 exists. The good match of the $K_{D I}$ value obtained in the second analysis step and $K_{D 1}$ derived by eq 4 indicates that not only the consecutive encapsulation of two $\mathrm{EH}^{+}$but also the direct $\left(\mathrm{EH}^{+}\right)_{2}-\mathrm{CB} 8$ formation with the interaction of $\left(\mathrm{EH}^{+}\right)_{2}$ and CB8 occur. The calculated binding constants are summarized in Table 1 .

NMR measurements in the presence of $190 \mu \mathrm{M}$ CB8 confirmed the inclusion complex formation. Higher CB8 concentration could not be employed because of the low solubility of CB8 even in the presence of $\mathrm{EH}^{+}$. The NMR spectra are displayed in Figure S4 in Supporting Information.

\subsection{Determination of the thermodynamic parameters of inclusion at high $\mathrm{EH}^{+}$concentration}

Isothermal calorimetric measurements at $\mathrm{pH} 4$ gave information on the thermodynamics of complex formation. Figure S2 in Supporting Information displays the experimental results for the titration of $124 \mu \mathrm{M} \mathrm{CB} 8$ to $8.0 \mu \mathrm{M} \mathrm{EH}{ }^{+}$solution at $298 \mathrm{~K}$. Exothermic complexation was observed. The data were consistent with a sequential binding to two sites model. To decrease the number of fitted parameters, $K_{11}$ and $K_{21}$, were taken from Table 1 and kept constant. The nonlinear least-squares analysis led to the thermodynamic quantities listed in Table 2. 
To verify the calorimetric results, the spectrophotometric titrations presented in Figure 5 were repeated at various temperatures. At each temperature the experimental data were fitted keeping $\mathrm{K}_{11}$ and $\mathrm{K}_{\mathrm{D}}$ fixed at the value calculated on the basis of the calorimetric determined enthalpy and entropy changes. $\Delta \mathrm{H}$ and $\Delta \mathrm{S}$ for the formation of $\mathrm{EH}^{+}-\mathrm{CB} 8$ is given in Table 2, whereas these quantities for $\mathrm{EH}^{+}$dimerization were taken from a recent paper (32). The van't Hoff plot of the calculated $\mathrm{K}_{21}$ binding constants is shown in Figure S3 in Supporting Information. The thermodynamic parameters derived therefrom are included in Table 2 . The results derived from fluorescence or spectrophotometric titrations agree, within the limits of experimental errors, with the data obtained by calorimetric measurements.

\section{Discussion}

Our results demonstrate that the binding of $\mathrm{EH}^{+}$both to $\mathrm{CB} 8$ and to $\mathrm{EH}^{+}-\mathrm{CB} 8$ are enthalpically driven processes. Despite the 38 -fold larger stability constant of the 1:1 complex, the inclusion of the first $\mathrm{EH}^{+}$is much less exothermic than the second binding step. Substantial entropy gain contributes to the driving force of $1: 1$ encapsulation, whereas $2: 1$ complexation is entropically highly unfavourable. The removal of high-energy water molecules from the apolar cavity of cucurbiturils was found to play a very important role in controlling the binding strength (33-36). The water network is moderately distorted in CB8 (34) and only a fraction of water is expelled by the inclusion of $\mathrm{EH}^{+}$. Therefore, $\mathrm{EH}^{+}-\mathrm{CB} 8$ formation is accompanied by a limited enthalpy diminution. The energy of the remnant water in $\mathrm{EH}^{+}-\mathrm{CB} 8$ becomes higher because less optimized interactions among the encapsulated water molecules can be developed. Consequently, the release of water upon embedment of the second $\mathrm{EH}^{+}$results in more substantial enthalpy gain than 1:1 complexation. The transfer of water from the interior of CB8 and from the hydrate shell of $\mathrm{EH}^{+}$to the bulk leads to entropy enhancement. The entropy loss 
due to the inclusion does not counterbalance this effect since the loose binding of $\mathrm{EH}^{+}$in the spacious CB8 causes relatively small entropy change. In contrast, $\mathrm{EH}^{+}$association with $\mathrm{EH}^{+}-\mathrm{CB} 8$ results in tightly packed complex, in which the degrees of freedom of the constituents are highly restricted. Hence, $\left(\mathrm{EH}^{+}\right)_{2}-\mathrm{CB} 8$ formation is accompanied by a significant entropy decrease. The considerably lower binding affinity of $\mathrm{EH}^{+}$to $\mathrm{EH}^{+}-\mathrm{CB} 8$ compared with the encapsulation in CB8 originates from the very unfavourable entropy contribution to the driving force in the former process.

$\mathrm{K}_{11}$ for $\mathrm{EH}^{+}-\mathrm{CB} 8$ formation is ca. 6-fold smaller than the corresponding quantity for the inclusion of berberine, an isoquinoline alkaloid, in CB8 (18). This difference arises from the slightly less negative $\Delta \mathrm{H}_{11}$ and the smaller entropy gain for $\mathrm{EH}^{+}$complexation. About 50-fold lower $\mathrm{K}_{21}$ value is obtained for $\left(\mathrm{EH}^{+}\right)_{2}-\mathrm{CB} 8$ (Table 1) compared to the analogous process of berberine.(18) Both $\Delta \mathrm{H}_{21}$ and $\Delta \mathrm{S}_{21}$ are significantly less negative in the case of $\mathrm{EH}^{+}$, and the enthalpy term dominates to a lesser extent when this alkaloid produces $2: 1$ complex. The binding affinity of $\mathrm{EH}^{+}-\mathrm{CB} 8$ to $\mathrm{CB} 8$ is low because of the electrostatic repulsion between the high electron density of oxygens at the portals of the two hosts. Much smaller driving force for 1:2 complexation compared with 1:1 association was also found when sanguinarine, a natural benzo[c]phenanthridine alkaloid, interacted with cucurbit[7]uril (17).

The results of the present study are in contrast to the conclusions of a former report on $\mathrm{EH}^{+}$confinement in $\mathrm{CB}$, which suggested only 1:1 complex formation (30). We found about 7.6-fold larger equilibrium constant for the 1:1 $\mathrm{EH}^{+}-\mathrm{CB} 8$ inclusion complex formation than the $2.1 \times 10^{5} \mathrm{M}^{-1}$ value published by Gavvala and coworkers (30). This discrepancy probably arises from the fact that the dimerization of $\mathrm{EH}^{+}(32)$ and the encapsulation of two $\mathrm{EH}^{+}$in the cavity of 
CB8 were not previously taken into account. The substantial variation of the fluorescence decay parameters is due to the change of the binding stoichiometry with host and guest concentrations.

\section{Supplementary Information}

Supplemental data for this article can be accessed http://

\section{Acknowledgement}

The authors very much appreciate the support of this work by the Hungarian Scientific Research Fund (OTKA, Grant K104201) and MedInProt Protein Science Research Synergy Program. Z. M. thanks the support of the János Bolyai Research Scholarship of the Hungarian Academy of Sciences.

\section{References}

(1) Walker, S.; Oun, R.; McInnes, F. J.; Wheate, N. J. Isr. J. Chem. 2011, 51, 616-624.

(2) Ghosh, I.; Nau, W. M. Adv. Drug Deliv. Rev. 2012, 64, 764-783.

(3) Shchepotina, E. G.; Pashkina, E. A.; Yakushenko, E. V.; Kozlov, V. A. Nanotechnologies in Russia 2011, 6, 773-779.

(4) Macartney, D. H. Isr. J. Chem. 2011, 51, 600-615.

(5) Ghale, G.; Ramalingam, V.; Urbach, A. R.; Nau, W. M. J. Am. Chem. Soc. 2011, 133, $7528-7535$

(6) Bailey, D. M.; Hennig, A.; Uzunova, V. D.; Nau, W. M. Chem. Eur. J. 2008, 14, 60696077.

(7) Megyesi, M.; Biczók, L.; Jablonkai, I. J. Phys. Chem. C 2008, 112, 3410-3416.

(8) Ghale, G.; Nau, W. M. Acc. Chem. Res. 2014, 47, 2150-2159. 
(9) Uzunova, V. D.; Cullinane, C.; Brix, K.; Nau, W. M.; Day, A. I. Org. Biomol. Chem. 2010, $8,2037-2042$.

(10) Montes-Navajas, P.; Gonzalez-Bejar, M.; Scaiano, J. C.; Garcia, H. Photochem. Photobiol. Sci. 2009, 8, 1743-1747.

(11) Hettiarachchi, G.; Nguyen, D.; Wu, J.; Lucas, D.; Ma, D.; Isaacs, L.; Briken, V. Plos One 2010, 5, e10514.

(12) Zhao, Y. J.; Pourgholami, M. H.; Morris, D. L.; Collins, J. G.; Day, A. I. Org. Biomol. Chem. 2010, 8, 3328-3337.

(13) Zhao, Y. J.; Buck, D. P.; Morris, D. L.; Pourgholami, M. H.; Day, A. I.; Collins, J. G. Org. Biomol. Chem. 2008, 6, 4509-4515.

(14) Wang, R. B.; Macartney, D. H. Org. Biomol. Chem. 2008, 6, 1955-1960.

(15) Saleh, N.; Koner, A. L.; Nau, W. M. Angew. Chem. Int. Ed. 2008, 47, 5398-5401.

(16) Appel, E. A.; Rowland, M. J.; Loh, X. J.; Heywood, R. M.; Watts, C.; Scherman, O. A. Chem. Commun. 2012, 48, 9843-9845.

(17) Miskolczy, Z.; Megyesi, M.; Tárkányi, G.; Mizsei, R.; Biczók, L. Org. Biomol. Chem. 2011, 9, 1061-1070.

(18) Miskolczy, Z.; Biczók, L. Phys. Chem. Chem. Phys. 2014, 16, 20147-20156.

(19) Miskolczy, Z.; Biczók, L. J. Phys. Chem. B 2014, 118, 2499-2505.

(20) Pandrangi, S.; Chikati, R.; Chauhan, P.; Kumar, C.; Banarji, A.; Saxena, S. Tumor Biol. 2014, 35, 723-737.

(21) Pohlit, A. M.; Rocha e Silva, L. F.; Henrique, M. C.; Montoia, A.; Amorim, R. C. N.; Nunomura, S. M.; Andrade-Neto, V. F. Phytomedicine 2012, 19, 1049.

(22) Stiborová, M.; Frei, E. Curr. Med. Chem. 2014, 21, 575-591.

(23) Liu, J.; Xiao, Y.; Allen, C. J. Pharm. Sci. 2004, 93, 132-143. 
(24) Fung, S. Y.; Yang, H.; Bhola, P. T.; Sadatmousavi, P.; Muzar, E.; Liu, M.; Chen, P. Adv. Funct. Mater. 2009, 19, 74-83.

(25) Dodin, G.; Schwaller, M.-A.; Aubard, J.; Paoletti, C. Eur. J. Biochem. 1988, 176, 371-376.

(26) Fung, S. Y.; Duhamel, J.; Chen, P. J. Phys. Chem. A 2006, 110, 11446-11454.

(27) Miskolczy, Z.; Biczók, L. J. Photochem. Photobiol. A: Chem. 2006, 182, 82-87.

(28) Miskolczy, Z.; Biczók, L.; Jablonkai, I. Chem. Phys. Lett. 2006, 427, 76-81.

(29) Miskolczy, Z.; Biczók, L.; Jablonkai, I. J. Phys. Chem. A 2012, 116, 899-900.

(30) Gavvala, K.; Sengupta, A.; Koninti, R. K.; Hazra, P. J. Phys. Chem. B 2013, 117, 1409914107.

(31) Megyesi, M.; Biczók, L. J. Phys. Chem. B 2010, 114, 2814-2819.

(32) Miskolczy, Z.; Biczók, L. Chem. Phys. Lett. 2016, 644, 292-295.

(33) Nau, W. M.; Florea, M.; Assaf, K. I. Isr. J. Chem. 2011, 51, 559-577.

(34) Biedermann, F.; Uzunova, V. D.; Scherman, O. A.; Nau, W. M.; De Simone, A. J. Am. Chem. Soc. 2012, 134, 15318-15323.

(35) Biedermann, F.; Vendruscolo, M.; Scherman, O. A.; De Simone, A.; Nau, W. M. J. Am. Chem. Soc. 2013, 135, 14879-14888.

(36) Biedermann, F.; Nau, W. M.; Schneider, H.-J. Angew. Chem. Int. Ed. 2014, 53, 1115811171. 